\title{
In Vitro Screening of a Culturable Soybean Cyst Nematode Cyst Mycobiome for Potential Biological Control Agents and Biopesticides
}

\author{
Deepak Haarith, ${ }^{1}$ Dong-gyu Kim, ${ }^{1}$ Noah B. Strom,${ }^{2}$ Senyu Chen, ${ }^{1}$ and Kathryn E. Bushley ${ }^{2, \dagger}$ \\ ${ }^{1}$ Department of Plant Pathology, University of Minnesota, St. Paul, MN 55108 \\ 2 Department of Plant and Microbial Biology, University of Minnesota, St. Paul, MN 55108 \\ Accepted for publication 6 April 2020.
}

\begin{abstract}
Fungal biological control of soybean cyst nematodes $(\mathrm{SCN})$ is an important component of integrated pest management for soybean. However, very few fungal biological control agents are available in the market. In this study, we have screened fungi previously isolated from SCN cysts over 3 years from a long-term crop rotation field experiment for their ability to antagonize SCN using (i) parasitism, (ii) egg hatch inhibition, and (iii) $\mathrm{J} 2$ mortality. We evaluated egg parasitism using an invitro egg parasitism bioassays and scored parasitism using the egg parasitic index (EPI) and fluorescent microscopy. The ability of these fungi to produce metabolites causing egg hatch inhibition and $\mathrm{J} 2$ mortality was assessed in bioassays using filter-sterilized culture filtrates.
\end{abstract}

ABSTRACT
We identified 10 high-performing isolates each for egg parasitism and toxicity toward SCN eggs and J2s and repeated the tests after storage for 1 year of cryopreservation at $-80^{\circ} \mathrm{C}$ to validate the durability of biocontrol potential of the chosen 20 isolates. Although the parasitic ability changed slightly for the majority of strains after cryopreservation, they still scored $5 / 10$ on EPI scales. There were no differences in the ability of fungi to produce antinemic metabolites after cryopreservation.

Keywords: biological control, bionematicide, disease control and pest management, fungi, Heterodera glycines, in vivo screening, mycobiome, nematodes, soybeans, soybean cyst nematode
Soybean cyst nematodes $(\mathrm{SCN})$ are the most economically consequential pest of soybeans in the United States (Koenning and Wrather 2010). The SCN reproduces at an extremely rapid rate. Within one growing season, even if only $3 \%$ of the available egg inoculum reproduces, the population of SCN may multiply 1,000 times (SCN Coalition 2016). The eggs within cysts produced during each growing season can accumulate and remain viable in soil for nearly a decade (Chen 2011; Koenning 2004), making eradication of the SCN from fields nearly impossible. These eggs and cysts are the main sources of inoculum for the disease. However, their populations can be managed to mitigate their economic impacts. Cultural practices such as rotating with a nonhost crop like corn, and genetic resistance have been the most important control methods for SCN in the field (Chen and Dickson 2012). Unfortunately, the most commonly deployed genetic resistance is losing effectiveness and new sources of genetic resistance are still being assessed for their potential to generate agronomically acceptable soybean varieties (Niblack et al. 2008). In this current scenario, there is a need for

†Corresponding author: K. E. Bushley; kbushley@umn.edu

Funding: This research was supported by U.S. Department of Agriculture-National Institute of Food and Agriculture (grant number NIFA 2015-67013-23419) and three one-year grants from the Minnesota Soybean Research and Promotion Council.

Author contributions: D.H. co-conceived and conducted the experiments, standardized production of axenic SCN cysts, co-created methods, methods figures, and authored the manuscript. D.K. helped D.H. with the experiments, cocreated methods figures, and developed fluorescent microscopy techniques and performed fluorescent microscopy of fungal egg parasitism. N.S. implemented the Unite clustering algorithm to cluster sequences. S.C. and K.E.B. are thesis advisers to D.H. and provided funding and guidance for the research and edited the manuscript.

*The $\boldsymbol{e}$-Xtra logo stands for "electronic extra" and indicates that two supplementary figures and one supplementary table are published online.

The author(s) declare no conflict of interest.

Copyright (C) 2020 The Author(s). This is an open access article distributed under the CC BY 4.0 International license. developing biological control as an important component of integrated nematode management.

Fungi are promising candidates for biological control as many of them can overwinter as spores in the soil, enabling their persistence in agroecosystems. As prolific producers of secondary metabolites with bioactivity against nematodes, they also represent an unexplored resource for novel bionematicides (Hallmann and Sikora 1996; Mousa and Raizada 2013). Natural antagonists of the SCN such as nematophagous fungi are promising candidates for biological control and are increasingly popular in organic production systems (Haarith et al. 2019). Nematophagous fungi have several modes of antagonizing nematodes which may target different stage of the nematode life cycle. Nematode trapping fungi produce special hyphal structures to trap vermiform nematodes in the soil, while others such as Hirsutella rhosilliensis and H. minnesotensis are endoparasites that also target the vermiform stage of the life cycle. Others directly parasitize SCN embryos or juveniles in eggs within the cysts or egg masses (Chen and Dickson 2012; Hu et al. 2018). All these nematophagous fungi have been shown to use direct parasitism, secretion of nematicidal metabolites, or both mechanisms to kill their prey (Chen et al. 1994, 2000a). Testing for these two mechanisms in vitro is a first step in screening for biological control potential in fungi.

The egg parasites of SCN, which destroy the source of new infective juveniles, are particularly attractive as potential biological control agents. An important aspect for a potential biocontrol fungus is the ability to grow well in artificial culture using limited nutrients to produce copious spores. The production of secreted metabolites with bioactivity against nematodes is another important mechanism. These metabolites may help the fungi weaken or paralyze their nematode prey and could also enable biocontrol fungi to be more competitive and counteract "fungistasis" or the inhibition by other microorganisms in the complex soil environment (Kerry 1988). Furthermore, these metabolites could also be developed into environmentally safe nematicides, even if the producing fungus is potentially pathogenic to plants. One of the few commercially available products from fungi, for example, is a bionematicide (DiTera; Valent BioSciences, Libertyville, IL) 
derived from the potential plant pathogen Myrothecium verrucaria (Warrior et al. 1999). Other desirable traits of candidate biocontrol agents needed to overcome bottlenecks to commercialization are important considerations (Fravel 1999; Whipps and Lumsden 2009). The ability to sporulate well to enable easy formulation and application methods, consistent efficacy, and retention of activity during transport and storage are the most significant traits to be considered in screening for biological control agents.

We recently characterized the SCN cyst mycobiome from SCN cysts isolated from a long-term soybean-corn rotation experiment in Waseca, Minnesota, U.S.A. (Haarith et al. 2019; Hu et al. 2018). In this study, we have screened this SCN cyst mycobiome for fungal isolates with the in vitro ability to (i) effectively parasitize SCN eggs, (ii) inhibit SCN eggs and juveniles with secreted metabolites, and (iii) grow quickly and make copious amounts of spores on artificial media. We have also developed a cost-effective and reliable fluorescence-based visualization of fungal-egg parasitism using a Calcofluor White M2R, a chitin-binding dye, and propidium iodide, a live-cell exclusion DNA binding dye to visualize invasion of SCN eggs by the fungal hyphae and cell death in the nematode embryo, respectively.

\section{MATERIALS AND METHODS}

Fungal isolates. All fungal strains were obtained from a longterm soy-corn rotation experiment at the University of Minnesota Southern Research and Outreach Station as detailed in Haarith et al. (2019). Morphologically similar fungi were grouped together into morphogroups. These morphogroups were then further divided into submorphogroups of five if the morphogroup contained numerous $(>5)$ members. One random isolate from each submorphogroup was selected as a morphotype for sequencing of the internal transcribed spacer 1 (ITS1) region for molecular identification. These morphotypes were identified to fungal genus based on BLAST hits of more than $97 \%$ similarity to their respective ITS fungal barcode gene in the NCBI nucleotide database. ITS sequences of all morphotypes were then clustered, using the USEARCH (Edgar 2010) run in QIIME (Caporaso et al. 2010), into clusters based on sequence similarity of $99 \%$ or more (Strom et al. 2019) and these were compared back to morphogroups to assess accuracy of the morphogroup classifications. Each cluster was also assigned a cluster representative strain by the USEARCH algorithm. All isolates were stored as mycelial plugs in $20 \%$ glycerol at $-80^{\circ} \mathrm{C}$ until needed for subsequent assays. A random representative of each cluster, or two to three representatives for clusters with more than five representatives, were tested from each USEARCH cluster, for their ability to grow on $1 / 4$ potato dextrose agar (PDA) from glycerol stock after the freeze-thaw. Clusters with only one representative were excluded from in vitro assays if the representative morphotype did not grow from glycerol stock.

Axenic SCN cysts. HG Type 0 SCN eggs were obtained from the nematology research laboratory at Southern Research and Outreach Center in Waseca and were rinsed thrice with autoclaved distilled water. The eggs were then decontaminated by incubating them at $25^{\circ} \mathrm{C}$ for $8 \mathrm{~h}$ in an aqueous solution of $100 \mathrm{ppm}$ each of streptomycin and chlorotetracycline and with Fungin (Invitrogen) at $50 \mu \mathrm{g} / \mathrm{ml}$ to remove any bacterial and fungal contaminants. The eggs were subsequently rinsed off the antibiotic solution using autoclaved distilled water and hatched in an aseptic environment with $4 \mathrm{mM} \mathrm{ZnCl} 2$ solution using mesh support and sterile coffee filters as described in a previous report (Chen and Liu 2005). Fresh second-stage juvenile (J2) hatchlings were filtered through sterile coffee filters into autoclaved distilled water. Hatchlings were then inoculated on 1-week-old susceptible soybean seedlings grown in 6-inch bleached cone-tainers filled with autoclaved 80:20 sand/soil mixture by applying $5 \mathrm{ml}$ of nematode suspension (at least 1,000/ $\mathrm{ml}$ ) in a $7 \mathrm{~cm}$ deep hole created parallel to the stem. The system was maintained in a clean growth chamber with a HEPA filter to filter out fungal spores under daily $14 \mathrm{~h}$ light at $27^{\circ} \mathrm{C}$ and $10 \mathrm{~h}$ dark at $22^{\circ} \mathrm{C}$, for 28 days postinoculation. The soybean plants were watered using autoclaved tap water and no fertilizers were used. The soil and roots were then washed to recover SCN cysts using a modified hand floatation and sucrose centrifugation described previously (Chen et al. 2001). These cysts were surface sterilized using $0.5 \% \mathrm{NaOCl}$ bleach solution for $3 \mathrm{~min}$ and decontaminated with antibiotics as discussed above. Cysts were assessed prior to each experiment to certify that no fungal or bacterial growth was observed when incubated on PDA and in PDB for 7 days at $25^{\circ} \mathrm{C}$.

Parasitism assay. Ten cysts were placed in a circle of $2.5 \mathrm{~cm}$ diameter on water agar. A $1-\mathrm{cm}$-square plug of a representative cluster isolate was inoculated at the center of this circle and incubated at $25^{\circ} \mathrm{C}$ until the fungal colony edge reached the cysts. After further incubation for 2 weeks at $25^{\circ} \mathrm{C}$, five random cysts were examined under a light microscope and an egg parasitic index (EPI) was assessed for each cyst based on the percentage of eggs colonized in each cyst (Chen et al. 1996; Chen and Chen 2002) on a scale of 0 to 10 ( 0 to $100 \%$ of eggs colonized). An average EPI was calculated for each representative fungal isolate (Supplementary Fig. 1A). Additional glycerol stocks were made for the top 10 highly parasitic fungal isolates and stored at $-80^{\circ} \mathrm{C}$. The parasitism assay was repeated for these 10 isolates from the newly prepared glycerol stocks after 1 year in storage.

Widefield fluorescence microscopy. The remaining five parasitized cysts from each water agar plate were collected by flooding the surface with autoclaved distilled water. The eggs within were released from their cysts using tissue pestles (Carolina) in 1.5-ml Eppendorf tubes, and simultaneously labeled with the fluorescent dyes propidium iodide (Invitrogen) and Calcofluor White M2R (Sigma-Aldrich). Several modifications were made to the manufacturers' protocols: for propidium iodide staining, RNase and antifade reagent treatments were not performed; for Calcofluor White M2R staining, samples were rinsed several times with $2 \times$ saline sodium citrate (SSC) medium following labeling. Samples were centrifuged at 2,655 $\times g$ RCF for 1 min after each staining step and the supernatants were removed. The stained cells were resuspended in $25 \mu \mathrm{l}$ of sterile distilled water; these are the labeled samples.

Widefield fluorescence microscopy of labeled samples was conducted at the University of Minnesota, University Imaging Centers (St. Paul, MN). Images were obtained using a C1144042U30 digital CMOS camera (Hamamatsu) on NIS-Elements Viewer (Nikon) through Z-stack of foci of interest using DAPI (excitation: $325 \sim 375 \mathrm{~nm}$, emission: $435 \sim 485 \mathrm{~nm}$ ) and DsRed (excitation: $590 \sim 650 \mathrm{~nm}$, emission: $590 \sim 650 \mathrm{~nm}$ ) filters for Calcofluor White $\mathrm{M} 2 \mathrm{R}$ and propidium iodide excitation, respectively. A DIC N2 LWD T-C Eclipse microscope condenser prism (Nikon) was used. Exposures were adjusted manually on a case-by-case basis to ensure visual clarity of microscopic features.

Grayscale tagged image file format (.tif/.tiff) files obtained through microscopy were analyzed using the Extended Depth of Field plugin algorithm on NIS-Elements Viewer to obtain a focused image. This focused image was processed on Photoshop CS5 (Adobe) for image processing. The Unsharp Mask filter was applied (amount: 150\%, radius: 3 pixels, threshold: 0 levels) to sharpen microscopic features. The levels tool was also manually adjusted for individual images to increase image contrast. Color corrections were made for Calcofluor White $\mathrm{M} 2 \mathrm{R}$ and propidium iodide images.

Hatch inhibition and $\mathbf{J} 2$ mortality assays. Fermentation and recovery of metabolites. All cluster representatives were incubated on Spezieller Nahrstoffarmer Agar (SNA; $1 \mathrm{~g}$ of $\mathrm{KH}_{2} \mathrm{PO}_{4}$, $1 \mathrm{~g}$ of $\mathrm{KNO}_{3}, 0.5 \mathrm{~g}$ of $\mathrm{MgSO}_{4} 7 \mathrm{H}_{2} 0,0.5 \mathrm{~g}$ of $\mathrm{KCl}, 0.2 \mathrm{~g}$ of glucose, $0.2 \mathrm{~g}$ of sucrose, and $20 \mathrm{~g}$ of agar per liter) at $25^{\circ} \mathrm{C}$ with $12 \mathrm{~h}$ light-dark cycles for 15 days. The plates were flooded with $4 \mathrm{ml}$ of sterile, ice cold $0.2 \%$ Tween 20 solution, and the solution was gently pipetted on the fungal colony four to five times. The Tween 
solution was then examined under a light microscope using a hemocytometer for the presence of fungal spores. Fungal isolates that did not produce any spores ( 95 isolates) were eliminated from this assay. Spores for each isolate $\left(10^{6}\right)$ were separately inoculated into $50 \mathrm{ml}$ of 1/4-strength Czapek-Dox broth (CDB) and incubated at $25^{\circ} \mathrm{C}$ for 15 days at $120 \mathrm{rpm}$. The flasks were then cooled overnight to $4^{\circ} \mathrm{C}$ and the contents were centrifuged at $2,500 \times g$ relative centrifugal force (RCF) for 15 min to separate the fungal hyphal biomass. The hyphal biomass was frozen at $-80^{\circ} \mathrm{C}$, while the supernatant was recovered and sterilized by passing through sterile surfactant free cellulose acetate (SFCA) syringe filters of 0.45 and $0.20 \mu \mathrm{m}$. Sterile supernatants were frozen at $-20^{\circ} \mathrm{C}$ until used for the assay (Supplementary Fig. S1B).

Hatch inhibition assay. Frozen supernatants were transferred to $4^{\circ} \mathrm{C}$ to thaw slowly for $24 \mathrm{~h}$ before the assay. Nylon membrane of $35 \mu \mathrm{m}$ pore size was supported on modified 1.5 -ml centrifuge tube tops to form a $\mathrm{J} 2$ permeable filter that fits a standard 24-well tissue culture plate well. These filters were autoclaved and used as supports for egg hatch. Five hundred SCN eggs were supported on a sterile filter that was suspended over $2 \mathrm{ml}$ of sterile supernatant in each of the 24 wells of the standard 24-well tissue culture plate and incubated at $27^{\circ} \mathrm{C}$ for $48 \mathrm{~h}$. The filters were then removed along with unhatched eggs and the number of J2s hatched was counted in each well (Supplementary Fig. 1B). The experiment was done with three replicates for each supernatant as well as for 1/4 CDB and autoclaved distilled water controls. An average percentage inhibition was calculated for the supernatant of each cluster representative isolate, as well as the media control using the following formula:

$$
\left(1-\frac{\text { Number of hatchlings in test solution }}{\text { Number of hatchlings in } \frac{1}{4} \mathrm{CDB}}\right) \times 100
$$

The hatch inhibition assay was repeated a second time for these 10 isolates 1 year later (2019) from cultures grown from glycerol stocks after 1 year in storage at $-80^{\circ} \mathrm{C}$.

$J 2$ mortality assay. Sterile supernatants of the 10 fungi showing the most effective egg hatch inhibition were also studied for their ability to reduce $\mathrm{J} 2$ viability. In a 24 -well plate setup, a suspension of $100 \mu \mathrm{l}$ containing 50 freshly hatched SCN J2s was added in each well with $1 \mathrm{ml}$ of supernatant and incubated at $25^{\circ} \mathrm{C}$ for 24 and $48 \mathrm{~h}$. $\mathrm{J} 2$ mortality was estimated under an inverted microscope, using $1 \mathrm{M}$ $\mathrm{NaOH}$ stimulation as described by Chen and Dickson (2000) (Supplementary Fig. 1B). Percentage mortality was calculated for all culture filtrates, media, and water controls. The experiment was conducted in triplicate.

Statistical analyses. The EPI and hatch inhibition data were analyzed each year (for each experiment run) using the formula "EPI (or) \%hatch inhibition i isolates" and isolates were grouped based on the least significant difference (LSD) method using 'agricolae' package in R (R Core Team 2013) and Bonferroni correction for $P$ values. For the $\mathrm{J} 2$ mortality assay, data from all six replicates were combined and analyzed using the formula "\% $\%$ mortality\% isolates" and grouped based on LSD like the other assays.

\section{RESULTS}

Fungal isolates and clustering. In a previous study, we examined 6,000 SCN cysts for fungal growth after surface sterilization. Fungi isolated from these cysts were grouped into morphogroups based on colony similarities on PDA. From each morphogroup, a random isolate for every five isolates in the morphogroup (morphotype) was selected for ITS sequencing and identification. ITS region amplification and subsequent Sanger sequencing was successful only for 1,540 morphotypes ( $94 \%$ success rate). The morphotypes were assigned to genus based on the first 20 BLAST hits (Haarith et al. 2019) and the chlor-tetracycline mycobiome was found to be dominated by 14 common genera (Haarith et al. 2019). For this study, the sequences of the 1,540 morphotypes were clustered into 326 operational taxonomic unit (OTU) clusters based on $99 \%$ or higher sequence similarity among their ITS regions using USEARCH. For the most part, all the representative morphotypes from a single morphogroup clustered together, providing support for their correct grouping based on morphology alone. Only four morphotype representatives (one from a morphogroup whose genus is Unknown, one identified as Endophyte, and two as Fusarium) clustered in two different clusters each (Supplementary Table S1). However, most of the genera identified were represented by more than one cluster, suggesting the clustering based on ITS was potentially able to distinguish groups below the genus level. Out of 326 clusters, 173 clusters had five or fewer members and a single morphotype representative, while the remaining 153 clusters had more than five members and at least two morphotype representatives (Supplementary Table S1). The most abundant genus, Fusarium, comprised a majority $(\sim 30 \%)$ of the isolates in the total mycobiome (Haarith et al. 2019) and was represented by 467 morphotypes that clustered into 28 clusters. The next most abundant genera, Ilyonectria, included 119 morphotypes found in 12 clusters; 106 morphotypes of Cylindrocarpon in 12 clusters; 64 morphotypes of Exophiala in 11 clusters; and 43 morphotypes of Clonostachys in 4 clusters (Supplementary Table S1).

In vitro screening approach. A total of 237 morphotype isolates representing 174 clusters successfully grew from the glycerol stocks. In the 174 clusters, several morphotypes from the same cluster successfully grew for the genera Fusarium, Ilyonectria, Cylindrocarpon, Leptosphaeria, and Mortierella. Those isolates that grew were tested through the workflow pipeline shown in Figure 1. As we considered ability to sporulate an important biocontrol trait, isolates that did not sporulate were not tested further. Those that did sporulate were tested in two different in vitro biocontrol assays for egg hatch inhibition or egg parasitism as detailed in Figure 1.

Egg parasitism assay. The EPI or the percentage of eggs colonized by the fungus in a cyst at 2 weeks from initial contact was expressed on a scale of 0 to 10 ( 0 to $100 \%$ colonization). The EPI of the 237 morphotypes belonging to 174 clusters were evaluated using light microscopy and the frequency distribution of EPI was calculated (Fig. 2A). About $83.54 \%$ (198) of all isolates tested had an EPI of 4 or less and were deemed to have low EPI; $10.12 \%$ (24) had a moderate EPI between 4.01 and 6; and $4.22 \%$ (10) had a high EPI between 6.01 and 8 . Only five morphotypes from five different clusters (three Fusarium, one Mortierella, and one Alternaria), which make up about $2 \%$ of all isolates tested, had very high EPI between 8.01 and 10, meaning 80.1 to $100 \%$ of all eggs in the cysts were colonized by those fungi within 2 weeks from initial contact. Three out of five of the isolates with very high EPI belonged to three different Fusarium clusters. However, the mean EPI of all F usarium isolates did not vary significantly from the mean EPI of other genera. Additionally, both very high and low EPI isolates were often found within the same cluster. The EPI of the most parasitic fungal isolates varied slightly between the two experiments conducted. Some of the 10 highly egg parasitic fungal isolates showed a slightly reduced EPI when tested from a secondary glycerol stock compared with the initial screen, but all still had an EPI above 4.5 (Fig. 2B). Light microscopy images of the representative cysts from each of the 10 highly egg parasitic fungi showed that the inoculated fungi had established itself in both the egg matrix and the eggs themselves (Supplementary Fig. S2). The control eggs incubated on water agar without a fungal plug were observed to be devoid of any colonization in contrast to those that were coincubated with the high EPI isolates.

Microscopic visualization of egg parasitism. In order to visualize fungi parasitizing SCN eggs and confirm that these are 
true egg parasites and not opportunistic saprobes of the cyst matrix or walls, we developed a fluorescent microscopy protocol to simultaneously stain fungal hyphae with Calcofluor White (blue) and apoptotic nuclei in the nematode eggs using propidium iodide fluorescence (red). Compared with the controls, which showed a diffuse signal for both dyes, the majority of eggs inoculated with fungi showed visible evidence of fungal hyphae penetrating eggs and brighter or more punctate signal for apoptotic cells within the nematode egg (Fig. 3). However, different fungal isolates showed different patterns of colonization. In some cases (isolates A303-17, C303-20, and D216-34), a dense network of fungal mycelia could be seen throughout the egg and replaced the J2s within the eggs. In contrast, a majority of eggs from cysts inoculated with isolate B21142 were empty at the end of the 2-week incubation period, when visualized using fluorescent microscopy. On the other hand, dead apoptotic cells of $\mathrm{J} 2 \mathrm{~s}$ inside eggs were observed in a majority of

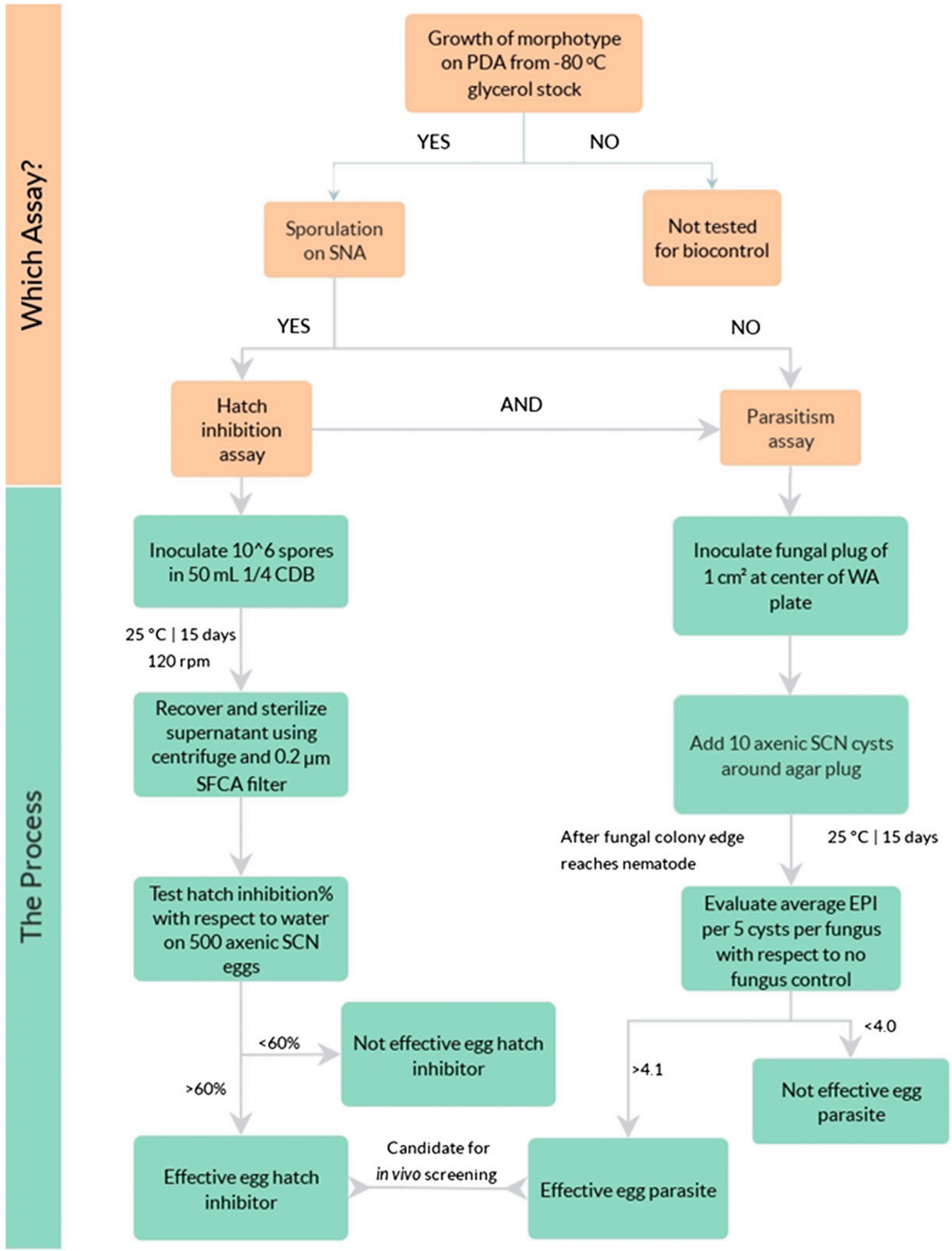

Fig. 1. Workflow for in vitro screening process to identify isolates with high parasitism, egg hatch inhibition, or juvenile (J2) toxicity. Only top 10 hatch inhibiting supernatants were tested for $\mathrm{J} 2$ mortality (not indicated in this flowchart). 
isolates, with particularly strong signals inside eggs that were inoculated with isolates A206-39, B209-6, D302-6, D407-12, and E308-24.

Egg hatch inhibition. Only 142 isolates out of the 237 morphotypes for which the EPI was evaluated produced spores and were subsequently assayed for hatch inhibition. Sterile culture filtrates from these isolates as well as a mock inoculated broth control were evaluated for their ability to inhibit SCN egg hatch.
About $35 \%$ of all isolates tested were able to inhibit egg hatch between 60.1 to $80 \%$ more than the $1 / 4$ CDB control. Seventeen percent of isolates had 80.1 to $100 \%$ more hatch inhibition compared with $1 / 4 \mathrm{CDB}$ control (Fig. 4A). Culture filtrates of isolate E403-35 had the highest inhibition of 95.68\%. On the other end of the spectrum, eight isolates stimulated better hatch compared with media control (E303-41, -213\%; D302-9, -68\%; B302-19, -34\%; E305-10, -32\%, E406-34, -10\%; A105-47 and
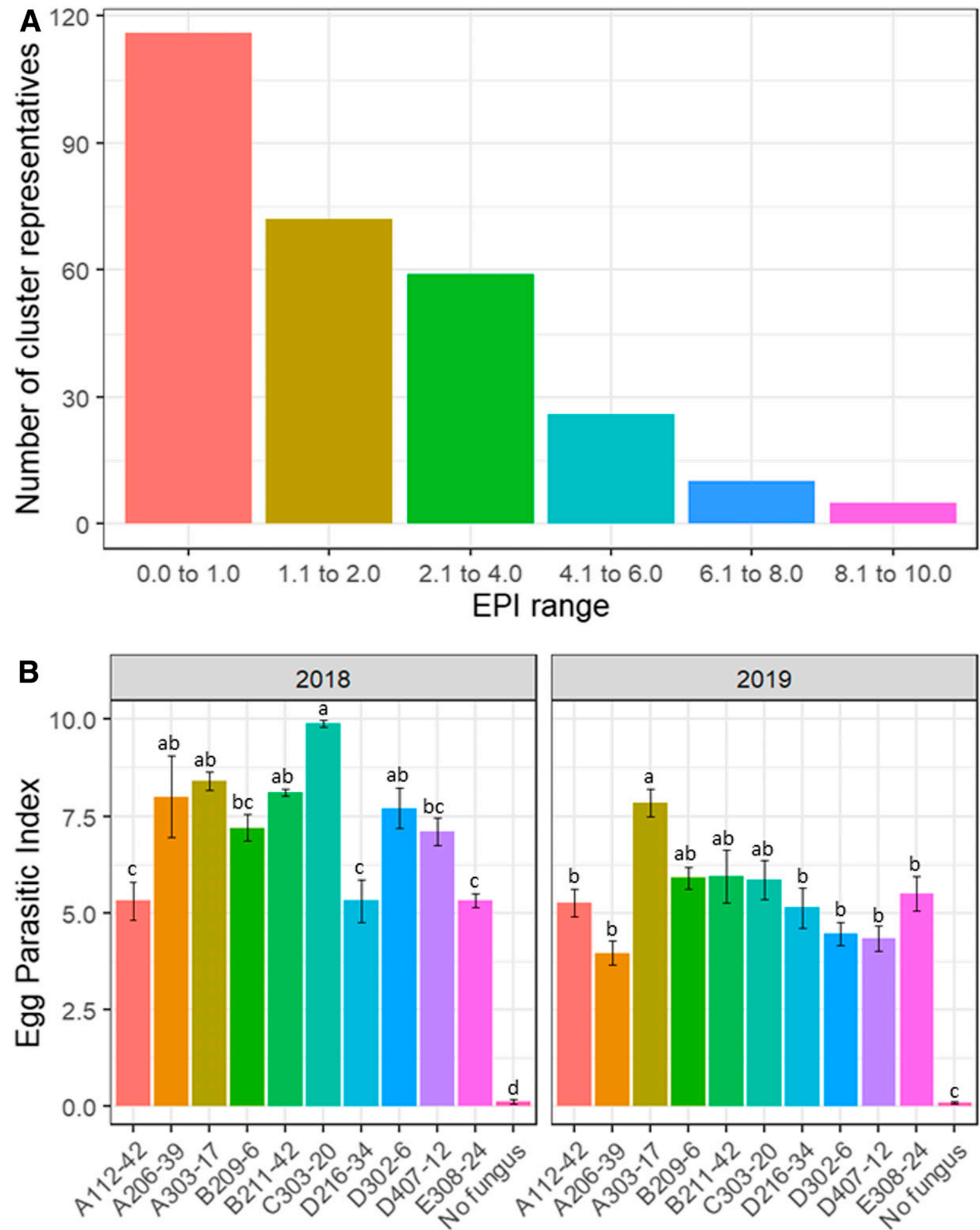

Fungal isolate

Fig. 2. A, Frequency distribution of egg parasitic index (EPI) of all fungi tested. B, EPI measurement for the 10 most highly parasitic fungal isolates estimated in 2018 after reviving cultures from an initial $-80^{\circ} \mathrm{C}$ glycerol stock and in 2019 after reviving from a secondary $-80^{\circ} \mathrm{C}$ glycerol stock. Significant groups were assigned based on least significant difference test within each year's data. Error bars indicate standard error. 
A112-40,-8\%; and D407-12,-4\%). Mock inoculated media alone inhibited egg hatch by about $35 \%$ compared with water controls. Therefore, only E303-41 and D302-9 filtrates could be considered as hatch stimulants. The inhibitory potentials of the culture filtrates for the top 10 isolates were significantly higher than mock-inoculated 1/4 CDB control (Fig. 4B), but they were not different from each other and are all well above $75 \%$ hatch inhibition. The ability of these isolates to produce nematicidal metabolites was not affected by the secondary glycerol cryopreservation (Fig. 4B).

J2 mortality. The 10 isolates with high potential for inhibiting SCN egg hatch also exhibited significant toxicity toward freshly hatched J2 (Fig. 5). At $24 \mathrm{~h}$ incubation, culture filtrates from isolate A302-17 was the most effective while there were no significant differences between the mortality rates of 1/4-strength Czapek-Dox broth and sterile deionized water. At $48 \mathrm{~h}$ incubation, isolates A216-
25, D302-43, E105-14, E403-35, and E413-17 had killed almost all the J2s. The 1/4 CDB medium control also showed higher mortality at $48 \mathrm{~h}$ compared with sterile deionized water. It is noteworthy that mortality in the culture filtrates of B306-45 and B411-6 was not different between 24 and $48 \mathrm{~h}$.

\section{DISCUSSION}

Fungi are promising candidates for biological control of the SCN, yet only a few studies have systematically screened the fungi found within SCN cysts for direct parasitism of eggs (Chen and Chen 2002, 2003; Chen et al. 1996, 2000b; Hu et al. 2018), inhibition of egg hatch and direct toxicity to J2s (Meyer et al. 1990; Mukhtar and Pervaz 2003; Nitao et al. 1999). In this study, we have used three techniques to identify potential biocontrol candidates. The fungal isolates screened in this study were isolated from a large number of
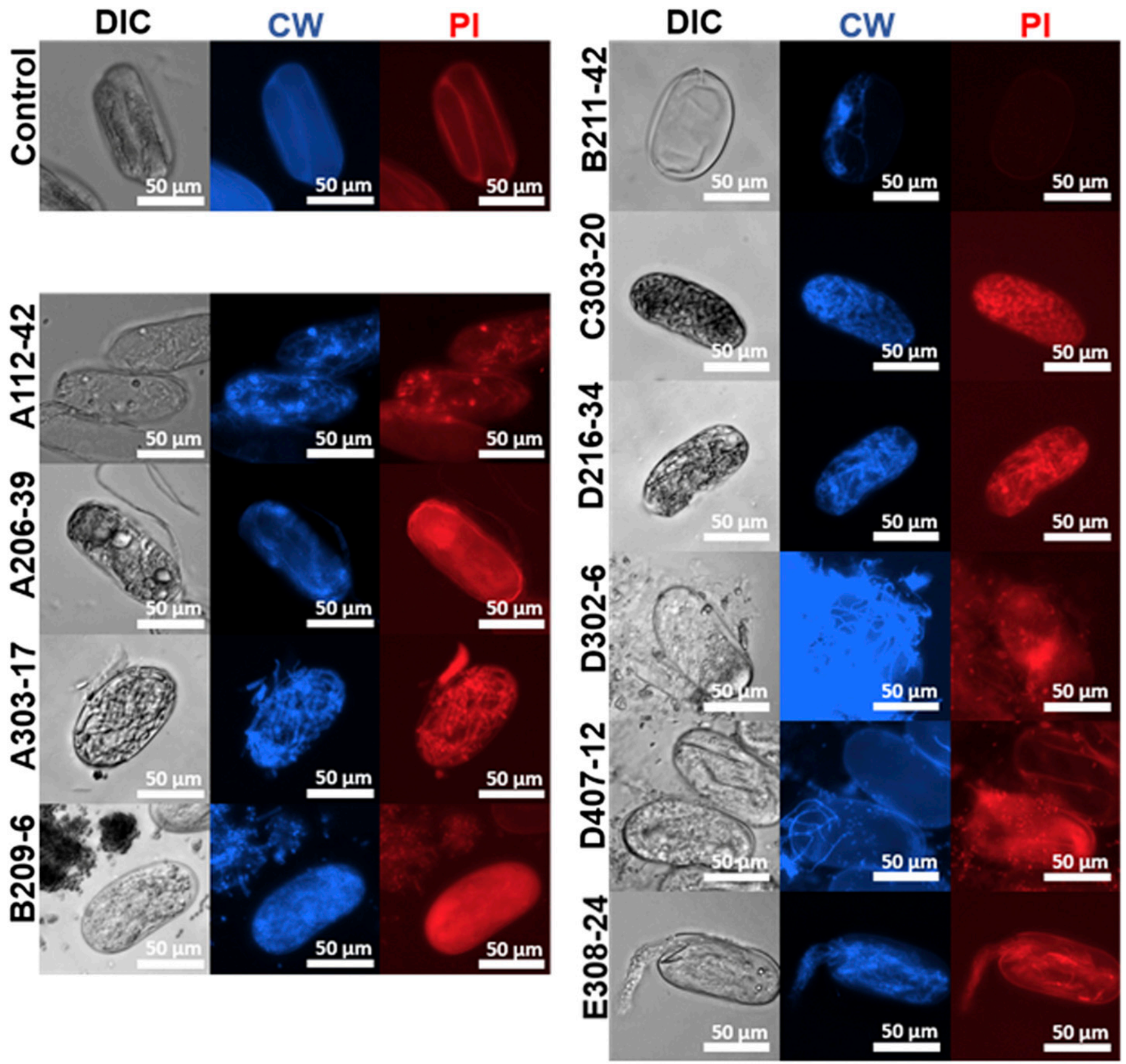

Fig. 3. Visualization of egg parasitism using differential interference contrast (DIC) and fluorescent microscopy. Calcofluor White (CW) binds chitin and traces the fungal hyphae. Propidium iodide (PI) fluoresces red when bound to active apoptotic nuclei. 
cysts $(6,000)$, therefore hopefully capturing most of the diversity of fungi inhabiting SCN cysts in this field. Here, we developed and implemented an efficient and high-throughput screening pipeline to assess the biocontrol potential within this SCN mycobiome. Isolates were first grouped based on colony morphology into morphogroups, then we sequenced the ITS region of a random representative morphotypes for every five members of a morphogroup, and these sequences were further clustered into 326 groups based on 99\% ITS sequence similarity (OTU clustering). Clustering corroborated groupings based on morphology and only a few isolates were sorted into two different clusters representing the same genera. Many genera were represented by several clusters (Supplementary Table S1). Fusarium and Ilyonectria, the most abundant genera in this mycobiome (Haarith et al. 2019), are both difficult to resolve into species using only the ITS region (Chaverri et al. 2011; O'Donnell et al. 2012). Therefore, clustering based on
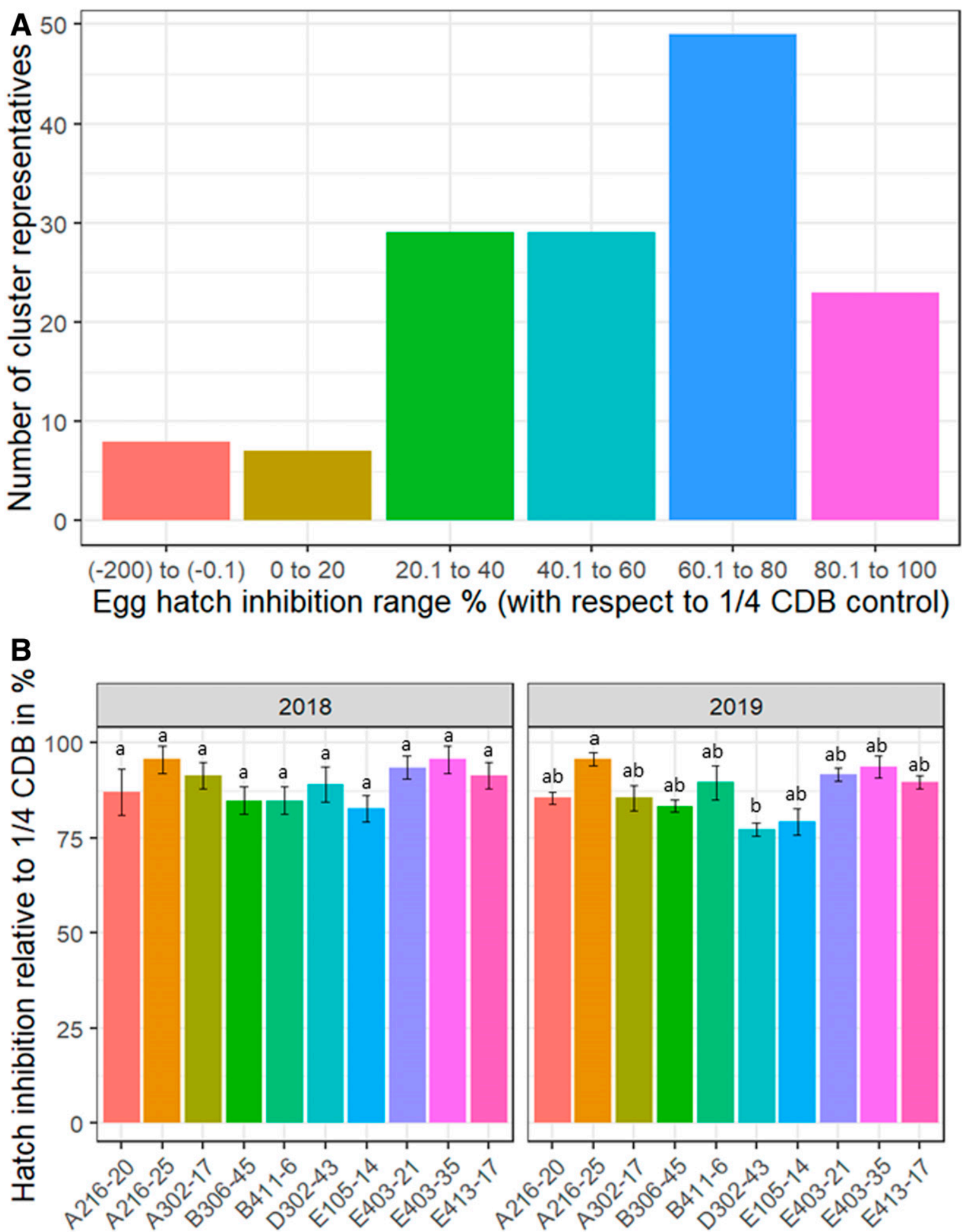

\section{Fungal isolate}

Fig. 4. A, Frequency distribution of hatch inhibition of all isolates screened. B, Hatch inhibition for the 10 highly inhibitory fungal isolates estimated in 2018 after reviving from an initial $-80^{\circ} \mathrm{C}$ glycerol stock and in 2019 after reviving from a secondary $-80^{\circ} \mathrm{C}$ glycerol stock. Significant groups were assigned based on least significant difference test within each year's data. Error bars indicate standard error. 
99\% ITS sequence similarity was used here primarily as a tool to narrow down our screening process and these clusters may or may not represent clusters at the species level. A total of 326 clusters were identified and we were able to screen representatives from over half of them (174 clusters). Unfortunately, we were not able to revive representatives from some clusters on 1/4 PDA after cryopreservation in glycerol at $-80^{\circ} \mathrm{C}$ and these were excluded from further screening. Not all fungi react the same way to a particular preservation technique (Ilyas and Soeka 2019; Morris et al. 1988). In fact, in a recent viability study done on preserving Beauveria bassiana strains, cryopreservation with glycerol consistently underperformed and reduced the fitness of the fungus (Oliveira et al. 2011). Due to the sheer number of isolates we had to handle, we only relied on one cryopreservation technique that is quite frequently used (Nakasone et al. 2004).

SCN cysts and eggs are the sedentary stages of the nematode and serve as the source of new inoculum and can remain viable in soil for nearly a decade (Chen 2011). Therefore, the ability to parasitize and/or kill SCN eggs is a beneficial trait for a biological control agent. Many fungi that can colonize cysts need not be good parasites of SCN eggs and might simply be commensals or saprobic (Carris et al. 1989; Chen et al. 2000a; Kerry 1988). Many studies have reported parasitic indices for fungi based on the EPI observed in cysts recovered from the field from which these fungi were isolated (Chen and Chen 2002, 2003; Hu et al. 2018). These approaches may not indicate the exact age of the cysts recovered nor the age of the relationship between the cyst and the associated fungus. It is impossible to deduce the age of the cysts and the contact time between the infected cysts and the fungal mycelia. However, different stages of cyst and eggs have been recovered and studied previously (Chen et al. 1994). Therefore, a good parasite might have a low EPI because it had less time of contact compared with a poor parasite that has had more time of contact with the cyst before they were collected from the field. Additionally, obligate fungi that cannot be cultured may be responsible for parasitism of eggs in the field, rather than the fungi that were isolated from the cyst. Considering the inherent limitations of EPI data from field, we used an in-vitro egg parasitism assay using axenic SCN cysts to directly evaluate fungal parasitism of SCN cysts and eggs. Previous studies showed that eggs at embryonic stages are more susceptible to fungal parasitism than the eggs in juvenile stages of development (Chen and Chen 2003). In this study, all cysts were collected at 30 days postinfection of soybean plants and were roughly of the same age. All fungi also had the same amount of contact time ( 2 weeks) with the cyst and eggs before their EPI was determined. $16.5 \%$ of all fungi tested had an EPI of 4 or higher, meaning that they could parasitize $40 \%$ or more of the eggs in the cysts within 2 weeks of mycelial contact. As discussed, the ability to maintain biocontrol activity during storage is an important consideration for biocontrol organisms. Therefore, we also evaluated the EPI of these 10 isolates with the highest EPI, after preserving them in glycerol at $-80^{\circ} \mathrm{C}$ for another year. Although the EPI was reduced for many isolates, it remained well above 4.5 , indicating these isolates retained their parasitic ability.

When tested from the initial glycerol stock, all the 10 most effective parasitic isolates had very high EPI. However, isolates A206-39, D302-6, C303-20, and D407-12 showed a decrease of about $30 \%$ in parasitism) when grown and tested from a secondary glycerol stock. Isolates A112-42, D216-34, and E308-24 retained their parasitism above $50 \%$ in both years, while others showed about a $10 \%$ decrease in parasitism. We subcultured these fungi in order to preserve them in glycerol at $-80^{\circ} \mathrm{C}$ for a secondary stock. Subculturing followed by storage in glycerol stock, revival on PDA and subsequent testing on WA could have made fungi lose some of their ability to infect nematodes and to become more saprobic. It has been shown previously that parasitic fungi could lose their virulence when subcultured on artificial media. The nematode parasitic fungus Drechmeria coniospora was observed to lose virulence with increasing number of subcultures (Zuckerman et al. 1989). It is also possible that the variation was due to different batches of SCN egg populations that were tested. However, in this study, the fungi revived from a secondary glycerol stock still retained an EPI of 4.5 or more, well above our initial cut-off of 4 index points, and could still be considered as effective SCN egg parasites. This study, like many other previous studies done on a variety of fungi, reveals the need to examine different preservation methods that could retain the parasitism of the cultures over long periods of time (Humber 1997; Nakasone et al. 2004; Sanchez-Pena and Thorvilson 1995; Zuckerman et al. 1989). Sporulation was another important criterion for our biocontrol screening. For commercial success of a biocontrol agent, spore formulations can be more easily stored and transported without losing viability (Parnell et al. 2016; Wraight et al. 2001). Spores are designed to withstand biotic stresses and can be desiccated easily to be used in seed coatings or as powders to be mixed in the fertilizer tanks of planters. Spores could also be suspended in irrigation water and easily applied near the roots where they are needed to combat nematodes.

Although light microscopy can help differentiate parasitized eggs from normal eggs with a developing embryo or juvenile stages, it is not adequate to observe the hyphal structures within individual eggs. In fact, significant amount of skill and training is necessary to tell apart eggs colonized by hyphae, eggs with under-developed embryo, and eggs that are apoptotic using light-microscopy. Fluorescence microscopy is hence an interesting tool to visualize the progression of parasitism as it could help differentiate fungal tissue from that of the nematode (Escudero and Lopez-Llorca 2012). Previous studies have used Calcofluor White M2R dye to visualize fungal features in their interactions with other organisms, including nematodes, because of its ability to bind chitin and $\beta$-glucans (Elad et al. 1983; Segers et al. 1994; Zavala-Gonzalez et al. 2015). This fluorescent dye binds nonspecifically to chitin and cellulose and fluoresces blue. In our egg parasitism experiments, since there were no plant materials, this dye bound to chitin in the cell wall of fungi and helped visualize hyphae. The outer layer of nematode eggs are made of vitelline and chitin is usually in the middle structural support layer (Bird and McClure 1976; Wharton 1980). Therefore, intact eggs do not bind with the calcofluor dye and only have a faint background calcofluor fluorescence as seen in our controls (Fig. 3). We also used a live-cell exclusion dye, propidium iodide, which nonspecifically intercalates with DNA of dead cells, to track cell death in the nematode within the parasitized eggs (Ferreira et al. 2015; Shapard et al. 2012). The combination of these two dyes helped us track fungal growth and parasitism in our assay by showing the extent of colonization by fungi in blue

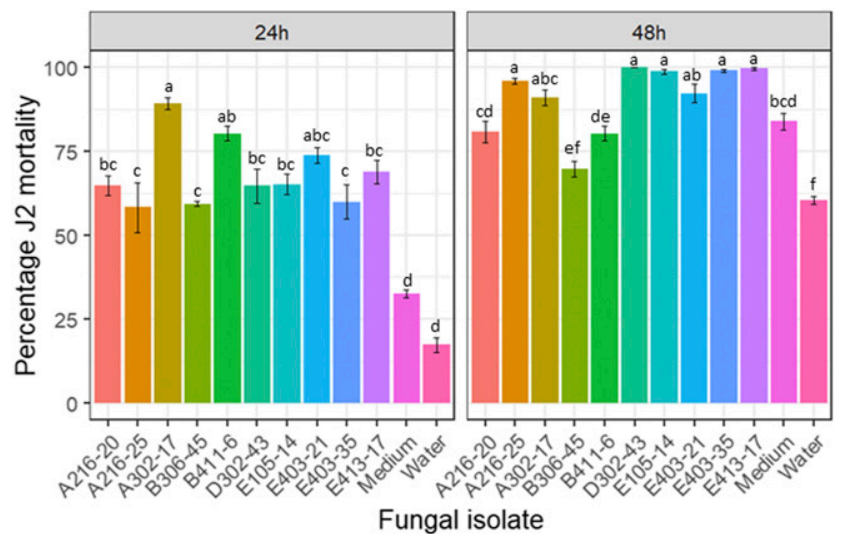

Fig. 5. Second-stage juvenile (J2) mortality estimates for the top 10 hatch inhibiting culture supernatants at 24 and $48 \mathrm{~h}$ time points. Mortality in 1/4 Czapek-Dox broth medium and water as reference. Significant groups were assigned based on least significant difference test. Error bars indicate standard errors. 
florescence and the resulting nematode death in red fluorescence. Thus, we have developed an affordable and rapid method to confirm egg parasitism by fungi as a part of this high-throughput screening of the mycobiome for biocontrol agents. With this technique, we were able to visualize some fungal isolates which colonized the egg and replaced the nematode embryo and juvenile within the egg entirely with hyphae, while others appeared to kill eggs without fungal penetration. Different fungal isolates also showed different levels of apoptosis in the juveniles within the egg, although almost all of them showed some level of apoptosis (Fig. 3). This could indicate either differences in the speed of parasitism or the mechanism (biotrophic versus necrotrophic) of parasitism, or both. Further focused investigations are necessary to ascertain this.

Fungi are known producers of several secondary metabolites and could employ both direct parasitism and indirect influence on nematodes using these metabolites. Consequently, several studies have tried to characterize the effect of fungal metabolites and culture filtrates against nematode hatching and/or juvenile mobility/ mortality. Not all fungi that were able to grow after cryopreservation could produce spores when incubated on a sporulation medium for 2 weeks. All fungi that did not sporulate by the end of 2 weeks were observed for 4 weeks until the medium dried out and only a few sporulated at the end of 4 weeks, but only sparsely. If we did not get enough spores to inoculate our $1 / 4 \mathrm{CDB}$ flasks $\left(10^{6}\right.$ spores in $\left.50 \mathrm{ml}\right)$, we did not evaluate that isolate for its ability to produce metabolites. Many studies have used hyphae or hyphal plugs to inoculate media in search of metabolites. A study that evaluated Verticillium lecanii (syn Lecanicillium lecanii) strains used mycelial agar plugs to inoculate liquid broth to study the production of nematotoxic metabolites (Shinya et al. 2008). This Verticillium study also looked at the effect of fungal metabolites on the development of fertile embryos into J2s and observed that some metabolites indeed affect nematode development. Most studies that investigate the effect of fungal metabolites on egg hatching incubate for 1 week or more (Massoud et al. 2002; Meyer et al. 2004; Nitao et al. 1999). However, our study was limited to 48-h incubation, which would only determine the effect of metabolites on hatch inhibition of mature eggs that are ready to hatch. Another study that investigated fungi in rhizosphere of plants for their ability to produce nematotoxic metabolites also inoculated fungal mycelial plugs (Qureshi et al. 2012). As the growth rate and biomass production per unit area for different fungi can vary, we chose to inoculate conidial spores, to control the inoculum levels and make comparisons on the metabolite production based on equal inoculum among the different isolates.

We used 1/4-strength CDB for secondary metabolite production for several reasons. It is a defined medium and is thus easier to evaluate active metabolites from other components in the media. The 1/4-strength medium helps more rapidly achieve carbon starvation, which can induce fungi to produce secondary metabolites (Nitsche et al. 2012). Among isolates that produced spores, the production of antagonistic metabolites was significantly more frequent in this mycobiome than direct parasitism. Fungi are known to produce secondary metabolites, some of which are thought to enable them to compete in soil and other environments with highly diverse or competitive microbial communities (Magan and Aldred 2008). The control 1/4 CDB medium also inhibited SCN egg hatch more than sterile deionized water, which is common for culture media. In a previous study that tested the effect of different media on metabolite production, a basal level of media antagonism was also observed for most media, often at levels greater than our medium (Nitao et al. 1999).

Unlike the EPI, the ability of fungi to produce antinemic metabolites did not diminish after subculturing and secondary cryopreservation. In addition to testing culture filtrates from egg parasitic fungi on egg hatch inhibition, we also tested the ability of the culture filtrates from the 10 most effective hatch inhibitors on J2 mortality. Many studies have previously investigated metabolite production as an important aspect of biological control. However, it is possible for metabolites to bind to sand and soil, reducing bioactivity when applied to soil. For example, flavipin was isolated from culture filtrate of a Chaetomium globosum strain using bioassay-guided fractionation. Although the compound had good in vitro effects, it did not inhibit nematodes when percolated through nematode-infected pots of plants (Nitao et al. 2002). Testing culture filtrates in planta on SCN-infested plants is an important next step in screening for bionematicides. In fact, as discussed, there is already a commercial biocontrol product in the market, DiTera, which is comprised of the dried culture filtrate of an isolate of Myrothecium verrucaria. This application method may be especially important if the culture filtrates have several nematotoxic compounds with synergistic or additive effect on each other (Bogner et al. 2016; Kundu et al. 2016).

In conclusions, we have used in silico and in vitro methods to perform high-throughput screening for candidate biocontrol agents of a large culturable mycobiome containing over 5,000 fungal isolates. Only a small percentage of fungi isolated from $\mathrm{SCN}$ cysts were strong parasites of SCN eggs, while a larger proportion were shown to produce compounds with bioactivity of egg hatch inhibition or toxicity toward $\mathbf{J} 2$. We identified 20 different sporulating candidate biological control agents, 10 each of high egg parasitic and high toxic metabolite producing isolates. We tested the reproducibility of their ability to parasitize SCN eggs or to inhibit SCN egg hatch in two separate experiments over 2 years after subculturing and cryopreservation. Future studies will evaluate these isolates in planta to evaluate their biocontrol potential on an SCN-susceptible soybean line. It is possible to find isolates of commercial interest from within these candidates based on both the in vitro and in vivo data.

\section{LITERATURE CITED}

Bird, A. F., and McClure, M. A. 1976. The tylenchid (Nematoda) egg shell: Structure, composition and permeability. Parasitology 72:19-28.

Bogner, C. W., Kamdem, R. S. T., Sichtermann, G., Matthäus, C., Hölscher, D., Popp, J., Proksch, P., Grundler, F. M. W., and Schouten, A. 2016. Bioactive secondary metabolites with multiple activities from a fungal endophyte. Microbiol. Biotechnol. 10:175-188.

Caporaso, J. G., Kuczynski, J., Stombaugh, J., Bittinger, K., Bushman, F. D., Costello, E. K., et al. 2010. QIIME allows analysis of high-throughput community sequencing data. Nat. Methods 7:335-336.

Carris, L. M., Glawe, D. A., Smyth, C. A., Edwards, D. I., Mycologia, S., and Feb, N. J. 1989. Fungi associated with populations of Heterodera glycines in two Illinois soybean fields. Mycologia 81:66-75.

Chaverri, P., Salgado, C., Hirooka, Y., Rossman, A. Y., and Samuels, G. J. 2011. Ascomycota and related genera with Cylindrocarpon-like anamorphs. Stud. Mycol. 68:57-78.

Chen, F., and Chen, S. 2002. Mycofloras in cysts, females, and eggs of the soybean cyst nematode in Minnesota. Appl. Soil Ecol. 19:35-50.

Chen, S. 2011. Soybean cyst nematode management guide. University of Minnesota Ext. 1-28. https://extension.umn.edu/soybean-pest-management/ soybean-cyst-nematode-management-guide

Chen, S., and Chen, F. 2003. Fungal parasitism of Heterodera glycines eggs as influenced by egg age and pre-colonization of cysts by other Fungi. J. Nematol. 35:271-277.

Chen, S., and Dickson, D. W. 2012. Biological control of plant-parasitic nematodes. Pages 761-811 in: Practical Plant Nematology. R. H. Manzanilla-Lopez and N. Marban-Mendoza, eds. Biblioteca Basica de Agricultura, Montecillo, Texcoco.

Chen, S., Dickson, D. W., Kimbrough, J. W., McSorley, R., and Mitchell, D. J. 1994. Fungi associated with females and cysts of Heterodera glycines in a Florida soybean field. J. Nematol. 26:296-303.

Chen, S., Dickson, D. W., and Mitchell, D. J. 1996. Pathogenicity of fungi to eggs of Heterodera glycines. J. Nematol. 28:148-158.

Chen, S., Dickson, D. W., and Mitchell, D. J. 2000a. Viability of Heterodera glycines exposed to fungal filtrates. J. Nematol. 32:190-197.

Chen, S., and Liu, X. 2005. Control of the soybean cyst nematode by the fungi Hirsutella rhossiliensis and Hirsutella minnesotensis in greenhouse studies. Biol. Control 32:208-219.

Chen, S., Liu, X. Z., and Chen, F. J. 2000b. Hirsutella minnesotensis sp. nov., a new pathogen of the soybean cyst nematode. Mycologia 92:819-824.

Chen, S., Porter, P. M., Reese, C. D., and Stienstra, W. C. 2001. Crop sequence effects on soybean cyst nematode and soybean and corn yields. Crop Sci. 41:1843-1849. 
Chen, S. Y., and Dickson, D. W. 2000. A technique for determining live second-stage juveniles of Heterodera glycines. J. Nematol. 32:117-121.

Edgar, R. C. 2010. Search and clustering orders of magnitude faster than BLAST. Bioinformatics 26:2460-2461.

Elad, Y., Chep, I., Boyle, P., and Henis, Y. 1983. Parasitism of Trichoderma spp. on Rhizoctonia solani and Sclerotium rolfsii-Scanning electron microscopy and fluorescence microscopy. Phytopathology 73:85-88.

Escudero, N., and Lopez-Llorca, L. V. 2012. Effects on plant growth and rootknot nematode infection of an endophytic GFP transformant of the nematophagous fungus Pochonia chlamydosporia. Symbiosis 57:33-42.

Ferreira, S. R., Antônio, T., Mendes, O., Bueno, L. L., De Araújo, J. V., Bartholomeu, D. C., and Fujiwara, R. T. 2015. A new methodology for evaluation of nematode viability. BioMed Res. Int. 2015:879263.

Fravel, D. 1999. Hurdles and bottlenecks on the road to biocontrol of plant pathogens. Australas. Plant Pathol. 28:53-56.

Haarith, D., Hu, W., Kim, D., Showalter, D. N., Chen, S., and Bushley, K. E. 2019. Culturable mycobiome of soya bean cyst nematode (Heterodera glycines) cysts from a long-term soya bean-corn rotation system is dominated by Fusarium. Fungal Ecol. 42:100857.

Hallmann, J., and Sikora, R. A. 1996. Toxicity of fungal endophyte secondary metabolites to plant parasitic nematodes and soil-borne plant pathogenic fungi. Eur. J. Plant Pathol. 102:155-162.

Hu, W., Strom, N., Haarith, D., Chen, S., and Bushley, K. E. 2018. Mycobiome of cysts of the soybean cyst nematode under long term crop rotation. Front. Microbiol. 9:386.

Humber, R. A. 1997. Fungi: Preservation of cultures. Pages 269-279 in: Manual of Techniques in Insect Pathology. L. A. Lacey, ed. Elsevier.

Ilyas, M., and Soeka, Y. S. 2019. Accelerated rate storage and viability test of Basidiomycetous fungal strains were cryopreserved at $-80^{\circ} \mathrm{C}$. Earth Environ. Sci. 308:012069.

Kerry, B. 1988. Fungal parasites of cyst nematodes. Agric. Ecosyst. Environ. 24:293-305.

Koenning, S. R. 2004. Population biology. Pages 73-88 in: Biology and Management of the Soybean Cyst Nematode. J. A. Wrather, D. P. Schmitt, and R. D.Riggs, eds. Schmitt \& Associates of Marceline, Marceline, MO.

Koenning, S. R., and Wrather, J. A. 2010. Suppression of soybean yield potential in the continental United States by plant diseases from 2006 to 2009. Plant Health Progress. https://doi.org/10.1094/PHP-2010-1122-01-RS

Kundu, A., Saha, S., Walia, S., and Dutta, T. K. 2016. Anti-nemic secondary metabolites produced by Fusarium oxysporum f. sp. ciceris. J. Asia Pac. Entomol. 19:631-636.

Magan, N., and Aldred, D. 2008. Environmental fluxes and fungal interactions: Maintaining a competitive edge. Chapter 2 in: Stress in Yeasts and Filamentous Fungi. P. van West, S. Avery, and M. Stratford, eds. Elsevier Ltd., Amsterdam, The Netherland.

Massoud, S., Meyer, S. L. F., Roberts, D., and Chitwood, D. 2002. Evaluation of Trichoderma virens and Burkholderia cepacia for antagonistic activity against root-knot nematode, Meloidogyne incognita. Nematology 2:871-879.

Meyer, S. L. F., Huettel, R. N., Liu, X. Z., Humber, R. A., Juba, J., and Nitao, J. K. 2004. Activity of fungal culture filtrates against soybean cyst nematode and root-knot nematode egg hatch and juvenile motility. Nematology 6:23-32.

Meyer, S. L. F., Huettel, R. N., and Sayre, R. M. 1990. Isolation of fungi from Heterodera glycines and in vitro bioassays for their antagonism to eggs. J. Nematol. 22:532-537.

Morris, G. J., Smith, D., and Coulson, G. E. 1988. A comparative study of the changes in the morphology of hyphae during freezing and viability upon thawing for twenty species of fungi. J. Gen. Microbiol. 134:2897-2906.

Mousa, W. K., and Raizada, M. N. 2013. The diversity of anti-microbial secondary metabolites produced by fungal endophytes: An interdisciplinary perspective. Front. Microbiol. 4:1-18.

Mukhtar, T., and Pervaz, I. 2003. In vitro evaluation of ovicidal and larvicidal effects of culture filtrate of Verticillium chlamydosporium against Meloidogyne javanica. Int. J. Agric. Biol. 5:576-579.

Nakasone, K. K., Peterson, S. W., and Jong, S. C. 2004. Preservation and distribution of fungal cultures. Pages 37-47 in: Biodiversity of Fungi: Inventory and Monitoring Methods. G. M. Mueller, G. F. Bills, and M. S. Foster, eds. Elsevier Academic Press, Burlington, MA.
Niblack, T. L., Colgrove, A. L., Colgrove, K., and Bond, J. P. 2008. Shift in virulence of soybean cyst nematode is associated with use of resistance from PI 88788. Plant Health Progress. https://doi.org/10.1094/PHP-20080118-01-RS

Nitao, J. K., Meyer, S. L. F., and Chitwood, D. J. 1999. In-vitro assays of Meloidogyne incognita and Heterodera glycines for detection of nematodeantagonistic fungal compounds. J. Nematol. 31:172-183.

Nitao, J. K., Meyer, S. L. F., Oliver, J. E., Schmidt, W. F., and Chitwood, D. J. 2002. Isolation of flavipin, a fungus compound antagonistic to plantparasitic nematodes. Nematology 4:55-63.

Nitsche, B. M., Jørgensen, T. R., Akeroyd, M., Meyer, V., and Ram, A. F. J. 2012. The carbon starvation response of Aspergillus niger during submerged cultivation: Insights from the transcriptome and secretome. BMC Genomics 13:380-403.

O’Donnell, K., Humber, R. A., Geiser, D. M., Kang, S., Park, B., Robert, V. A. R. G., et al. 2012. Phylogenetic diversity of insecticolous fusaria inferred from multilocus DNA sequence data and their molecular identification via FUSARIUM-ID and Fusarium MLST. Mycologia 104:427-445.

Oliveira, I., Pereira, J. A., Bento, A., and Baptista, P. 2011. Viability of Beauveria bassiana isolates after storage under several preservation methods. Ann. Microbiol. 61:339-344.

Parnell, J. J., Berka, R., Young, H. A., Sturino, J. M., Kang, Y., Barnhart, D. M., et al. 2016. From the lab to the farm: An industrial perspective of plant beneficial microorganisms. Front. Plant Sci. 7:1-12.

Qureshi, S. A., Ruqqia, Sultana, V., Ara, J., and Ehteshamul-Haque, S. 2012. Nematicidal potential of culture filtrates of soil fungi associated with rhizosphere and rhizoplane of cultivated and wild plants. Pak. J. Bot. 44:1041-1046.

R Core Team. 2013. R: A language and environment for statistical computing. R Foundation for Statistical Computing, Vienna.

Sanchez-Pena, S. R., and Thorvilson, H. G. 1995. Effect of long-term cryogenic storage and conidial suspending agents on the virulence of Beauvaria bassiana toward Solenopsis invicta. J. Invertebr. Pathol. 65:248-252.

SCN Coalition. 2016. What you need to test your fields to know your numbers. The SCN Coalition. https://www.thescncoalition.com/application/files/ 4715/1871/9690/why-test-your-fields.pdf

Segers, R., Butt, T. M., Kerry, B. R., and Peberdy, J. F. 1994. The nematophagous fungus Verticillium chlamydosporium produces a chymoelastaselike protease which hydrolyses host nematode proteins in situ. Microbiology 140:2715-2723.

Shapard, E. J., Moss, A. S., and San Francisco, M. J. 2012. Batrachochytrium dendrobatidis can infect and cause mortality in the nematode Caenorhabditis elegans. Mycopathologia 173:121-126.

Shinya, R., Aiuchi, D., Kushida, A., Tani, M., Kuramochi, K., and Koike, M. 2008. Effects of fungal culture filtrates of Verticillium lecanii (Lecanicillium spp.) hybrid strains on Heterodera glycines eggs and juveniles. J. Invertebr. Pathol. 97:291-297.

Strom, N., Hu, W., Haarith, D., Chen, S., and Bushley, K. 2019. Corn and soybean host root endophytic fungi with toxicity towards the soybean cyst nematode. Phytopathology 110:603-614. https://doi.org/10.1094/PHYTO-07-19-0243-R

Warrior, P., Rehberger, L. A., Beach, M., Grau, P. A., Kirfman, G. W., and Conley, J. M. 1999. Commercial development and introduction of DiTera, a new nematicide. Pestic. Sci. 55:376-379.

Wharton, D. 1980. Nematode egg-shells. Parasitology 81:447-463.

Whipps, J. M., and Lumsden, R. D. 2009. Commercial use of Fungi as plant disease biological control agents: Status and prospects. Pages 9-22 in: Fungi as Biocontrol Agents: Progress, Problems and Potential. T. M. Butt, C. W. Jackson, and N. Magan, eds. CABI Publishing, Wallingford, U.K.

Wraight, S. P., Jackson, M. A., and de Kock, S. L. 2001. Production, stabilization and formulation of fungal biocontrol agents. Pages 253-285 in: Fungi as Biocontrol Agents: Progress, Problems and Potential. T. M. Butt, C. Jackson, and N. Magan, eds. CABI Publishing, Wallingford, U.K.

Zavala-Gonzalez, E. A., Escudero, N., Lopez-Moya, F., Aranda-Martinez, A., Exposito, A., Ricano-Rodriguez, J., et al. 2015. Some isolates of the nematophagous fungus Pochonia chlamydosporia promote root growth and reduce flowering. Ann. Appl. Biol. 166:472-483.

Zuckerman, B. M., Dicklow, M. B., Coles, G. C., and Marban-Mendoza, N. 1989. Loss of virulence of the endoparasitic fungus Drechmeria coniospora in culture. J. Nematol. 21:135-137. 